\title{
The improved automatic control points computation for the acoustic noise level audits
}

\author{
Tomáš Drábek ${ }^{1}$, Jan Holub ${ }^{1}$ \\ ${ }^{1}$ Czech Technical University in Prague, Faculty of Electrical Engineering, Dept. of Measurement, Technicka 2, 16627 Prague 6, Czechia
}

\begin{abstract}
The acoustic noise level in the interior is one of the quantities specified by a standard and is subject to audits to ensure a comfortable living environment. Currently, the noise level audits are performed manually by a skilled operator, who evaluates the floor plan and uses it to calculate the control points location in which the measurement is performed. The computation is proposed to automate the audit by formulating an optimisation problem for which an algorithm was designed. The algorithm computes the solution that satisfies all constraints specified in the standard, for example, the minimum distance among the control points and fixed obstacles (walls or columns). In the proposed optimisation problem, the fitness function was designed based on the measurement purpose, and two typical use-cases were analysed: (i) long-term stationary noise measurement and (ii) recurring short-term noise measurement. Although the set of control points for both use cases complies with the given standard, it is beneficial to distinguish the location of control points based on the measurement purpose. The number of control points is maximised for the stationary noise and for the immediate coverage area for the short-term noise. The proposed algorithms were tested in a simulation for several floor plans of different complexity.
\end{abstract}

Section: RESEARCH PAPER

Keywords: Noise; measurement; algorithms; automation; determination of control points

Citation: Tomáš Drábek, Jan Holub, The improved automatic control points computation for the acoustic noise level audits, Acta IMEKO, vol. 10, no. 3, article 15, September 2021, identifier: IMEKO-ACTA-10 (2021)-03-15

Section Editor: Lorenzo Ciani, University of Florence, Italy

Received February 7, 2021; In final form August 6, 2021; Published September 2021

Copyright: This is an open-access article distributed under the terms of the Creative Commons Attribution 3.0 License, which permits unrestricted use, distribution, and reproduction in any medium, provided the original author and source are credited.

Corresponding author: Tomáš Drábek, e-mail: tomas.drabek@fel.cvut.cz

\section{INTRODUCTION}

The acoustic noise level is measured in human-occupied buildings to ensure comfortable living conditions. As described in [1], temperature, humidity, and $\mathrm{CO}_{2}$ concentration are the usually monitored indoor quantities. Measurement of the acoustic noise level, together with the mentioned quantities, can be used to improve the quality of indoor living. The noise measurement process is described in national standards that specify, for example, restrictions for placing control points, the duration of the individual measurements, or measurement device specifications. National supervisory authorities or private companies use these standards to determine noise levels both indoors and outdoors. Based on the measured values, they provide final recommendations. The whole process of measuring noise is time-consuming. Therefore, it is advisable to automate at least some steps of the process [2]. This paper aims to design automatic algorithms for placing the control points into a given room by obeying all constraints set by the standard. The measurement of the noise level is then performed in these control points.

The international and national standard [3], [4], for which the proposed method is designed, does not distinguish different measurement purposes and specifies only general rules for the location. In this article, two measurement purposes are proposed distinguishing: (i) living condition verification for a long-term stationary noise and (ii) living condition verification for a shortterm recurring noise. For the former, the algorithm can plan for resource allocation as the noise is presented continuously. Therefore, the algorithm aims at covering the room with as many control points as possible at the end of the measurement. For the latter, the resource allocation cannot be planned as the period of the noise signal is not known as a priory. The proposed algorithms place the control points to cover the maximum area in the limited time, in this case, at each iteration. These two different criteria result in different control points placing strategies, as depicted in Figure 1.

The current measurement procedure is performed by measuring noise levels in a network of control points. The control points are distributed around the room based on the 
restrictions given by the standard, and a qualified operator determines their density and positions. The distance of adjacent control points must be no less than $0.7 \mathrm{~m}$, and at least one control point must be located in a corner. In addition, all points must be at least $0.5 \mathrm{~m}$ away from the wall and at least $1 \mathrm{~m}$ away from significantly sound-transmitting elements such as windows or entrance openings for air supply. Windows and doors must be closed during measurement. The location of the measuring points defined in this way is determined manually by the operator according to the room's dimensions. Noise measurements are then made at these points at a height between $1.2 \mathrm{~m}$ and $1.5 \mathrm{~m}$ from the ground. The measuring instrument is directed towards the source of the incoming noise or vertically upwards if the direction of the noise source is not defined. A certified sound level meter is used as the measuring instrument.

Several articles have been published to measure and reduce noise. Some publications focus on noise caused by equipment (aircraft, cars, turbines). The article [5] dealt with the possibility of measuring aircraft noise using linear microphone arrays. Using this method, an undistorted record of aircraft noise was achieved.

The article [6] also deals with determining aircraft noise and selecting this noise from background noise. Against previous research, they use neural networks to recognise only aircraft noise. The accuracy converged to $99.84 \%$.

The publication [7] deals with the measurement of noise in landing aircraft that use thrust reversers to slow down aircraft after landing at Madrid-Barajas Airport. The paper presents possible improvements for detecting noise from the reverse thrust and the direction of incoming noise.

Another sector where noise is measured is the cars industry. The article [8] presents and verifies the application of Statistical Energy Analysis (SEA). The application is used for 3D modelling of noise reduction in the interior of a car from the drivetrain. Part of the work is also a proposal of measures to reduce noise.

Wind turbines are a separate branch of noise measurement. The standards specify, for example, noise measurement methods, requirements for measuring instruments, evaluation. Standard [9] provides overall wind turbine noise measurement standards. Conversely, standard [10] focuses more on the aeroacoustics noise of wind turbines. For example, the article [11] deals with noise measurement in the interior of buildings close to wind farms.

Another approach to noise measurement requires involving citizens and using their smart devices to monitor noise in their immediate vicinity. Such a measurement was dealt with in a study [12] which, using this method, created spatial and temporal maps of noise.

Noise measurements are also often performed indoors. In [13], the interior noise reduction index (NRI) was determined. The article deals with the definition of the index for NRIs with open windows for the summer months. A theoretical model was created and compared with experimentally obtained data.

Today, several software programs simulate acoustic conditions in buildings. Based on the created model of rooms with specified noise sources, technicians can create a noise map. In [15], the authors simulated a noise map and used it to identify critical areas using reference measurements and RAP-ONE software (Room Acoustics Prediction and Occupational Noise Exposure).

Article [16] deals with the measurement of noise at the place of residence of 44 schoolchildren. The measurements were performed in the children's rooms and in the room where the

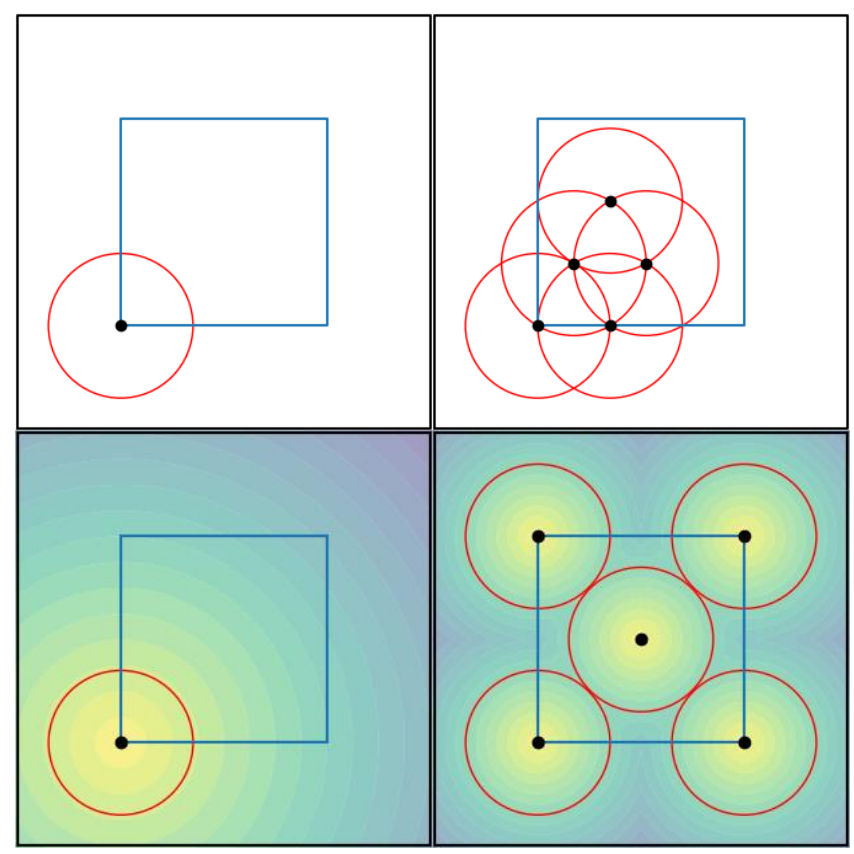

Figure 1. The control points location (black dots) for stationary noise (top row) and short-term recurring noise (bottom row) measurement. The left figures show the first iteration of both processes, in which the control point is located to the corner of the polygon defined by a $1 \mathrm{~m}$ distance from walls. The algorithm for stationary noise measurement (top) places control points step-by-step to put as many control points as possible while not placing them closer than the minimum distance visualised by the red circle. On the other hand, the short-term recurring noise spread points quickly leading to broad coverage in a short time by minimising the objective function visualised by the contour plot. Therefore, the total number of control points that can fit into the room is smaller for short-term recurring noise.

schoolchildren spent most of their time. Outdoor noise was also recorded during the measurement.

The sound pressure level affects the workplace and, for example, medical facilities where patients are treated. Article [17] deals with the measurement of noise around and inside the hospital. A total of 24 measurements were performed on the outer facade of the hospital and 21 measurements inside. From the measured data, it was evident that they exceeded the set limit.

The difference between outdoor and indoor noise is also described in the article [18]. The study performed noise measurements inside and outside the building at 102 inhabitants, with open, closed or semi-open windows playing a significant role. The result of the study was the creation of a statistical model that can be used to estimate the sound exposure inside the building.

Noise measurement using a robotic unit is becoming more common these days. In paper [19], the humanoid robotic unit was equipped with, among other sensors, a sound level meter. The humanoid measured values at 20 points in the room. Robot evaluated the comfort for the room based on an interaction with a human operator and from the measured values.

The article [20] on noise maps outside buildings also used an autonomous mobile robotic platform equipped with a sound level meter to measure noise. The measured values by the robot were compared with a model of known sources and with manual measurement. In conclusion, it was stated that noise maps should be based on the application and the environment.

All the publications mentioned above focused on the processing of the noise measurement data. On the other hand, the present paper aims to show the possibility of automating 
determining control points for measuring indoor noise. The novel contribution of this article is in the noise measurement methodology consisting of the design and testing of both purposes mentioned above, living condition verification for a long-term stationary noise and living condition verification for a short-term recurring noise. The control points are determined to meet the conditions based on the standard.

The algorithms for calculating control points in a room are based on the assumption that they do not have previous information about the room's parameters besides the floor plan. Therefore, individual elements (windows, openings in the wall, etc.) cannot be identified. For this reason, the condition is set that the control points are located $1 \mathrm{~m}$ from the wall. This condition is based on the requirement for distance from significantly sound-transmitting elements. All the above distances satisfy this condition.

The article is divided into three parts. Section 2 deals with the description of proposed noise measurement solution for the two considered purposes. Section 3 adresses the validation of the functionality of the proposed solution. Finally, the conclusions are summarised in Section 4.

\section{PROPOSED APPROACH}

Indoor noise measurements are performed at control points. The set of control points will be marked with the symbol $\chi$ and the individual control points with the symbol $x$ :

$\chi=\left\{x_{1}, x_{2}, \ldots, x_{n}\right\}$

where $n$ is the number of control points.

First, the area where the measurements will be performed is defined. The area of the room is denoted as $P$, and the wall of the room (boundary) represents the polygon $\partial P$. Then the control points will be located in the inner area $I$ defined as:

$I=\{x \mid x \in P \wedge d(x, \partial P) \geq 1\}$

All control points $x_{n}$ are located in the inner area of the room I:

$x_{n} \in I$.

The standard specifies the minimum requirement for the mutual Euclidean distance of control points, and it is $0.7 \mathrm{~m}$ :

$d\left(x_{i}, x_{j}\right) \geq 0.7$,

where $x_{i}$ a $x_{j}$ are control points, and indices take values:

$i, j \in\{1, \ldots, n\} ; i \neq j$.

According to the above standard, there should be at least one control point in the corner of the room. A corner is defined as a point that makes an angle below $180^{\circ}$ between two lines representing the walls of a room. The corners between $60^{\circ}$ and $120^{\circ}$ have priority in the selection. The corner(s) with the nearest angle to $90^{\circ}$ is selected if there is no corner in this range. The above parameters are shown in Figure 2.

Manually determining and setting control points is timeconsuming, hence this process was decided to automate two specific noise measurement purposes. The first is a long-term stationary noise, and the second is a regular short-term noise. Software algorithms were created for both of these purposes.

\subsection{Long-term stationary noise}

Long-term stationary noise is caused, for example, by operation from a factory in the neighbourhood or on a construction site. At the measuring point, the measurement takes place for an unnecessary time to ensure a sufficiently long and

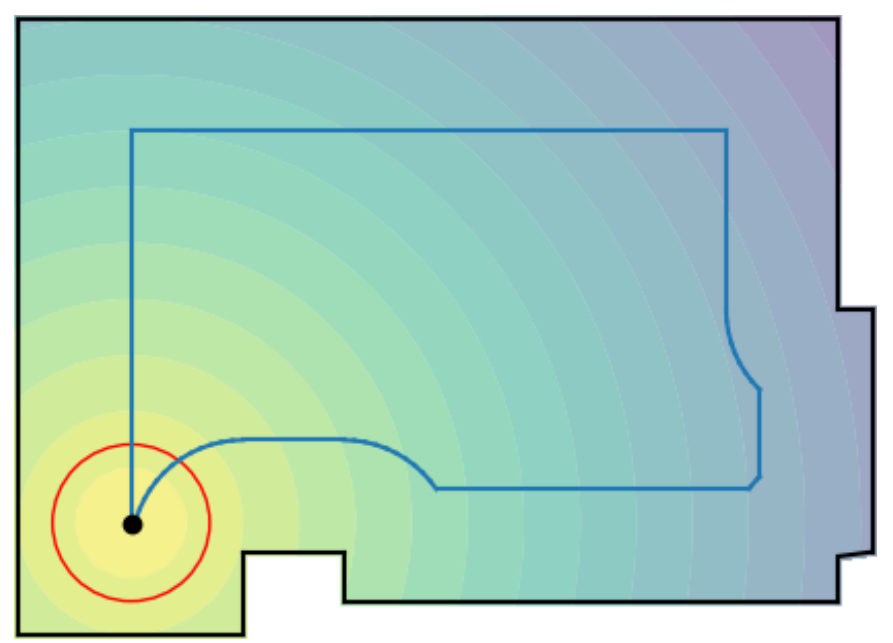

Figure 2. The walls of the room (black lines) represent the boundary of the polygon $\partial P$, where $P$ represents the area of the room. The blue lines delimit the measuring area that is at a $1 \mathrm{~m}$ distance from $\partial P$. The colored background of the room is used only in the algorithm for regular short-term noise, where it shows the distance from each already determined control point in space. At the control point, the background brightens and darkens with increasing distance. This illustration is used for regular short-term noise when it is appropriate to find the farthest control point in the defined area from already specified control points. The color background is recalculated each time iteration.

high-quality noise measurement. The measurement at the given control point has been declared demonstrable. After this interval, the measuring apparatus is moved to a new control point for further recording. The proposed algorithm searches for the maximum set of control points according to the specified parameters for maximum noise capture in a given space. We are looking for a maximum set that meets the following conditions:

$\chi^{*}=\max |\chi|$,

where $\chi^{*}$ is the maximum number of control points in a given room, and $|\chi|$ is the number of control points for the set $\chi$.

The entire algorithm was designed to obtain the maximum number of control points in area $I$. Obtaining data from the maximum number of control points would provide a more accurate map of capturing noise levels in space. The algorithm works by creating a list of all corners in the measured area $I$ (equation 2). It then passes through the individual corners and determines two intersections at a distance of $0.7 \mathrm{~m}$ from the given corner with $\partial I$. Thus, the algorithm obtains at least two initial variants for each corner. The program uses a recursive algorithm for each initial variant and creates a list of control points for each such recursion by following these steps:

1) From the already determined points, it draws a circle with a radius of $0.7 \mathrm{~m}$. The intersections with other circles give new control points.

2) If such an intersection does not exist and an intersection with $\partial I$, it places a control point at this intersection.

3) If the algorithm does not find any new control point in the iteration, it terminates the recursion and saves the total number (list) of found control points for the given variant.

The algorithm provides the list of the best results set - the maximal list of control points.

\subsection{Short-term recurring noise}

This is a noise caused, for example, by public transport (trams, buses) at the place of residence, workplace or school. 


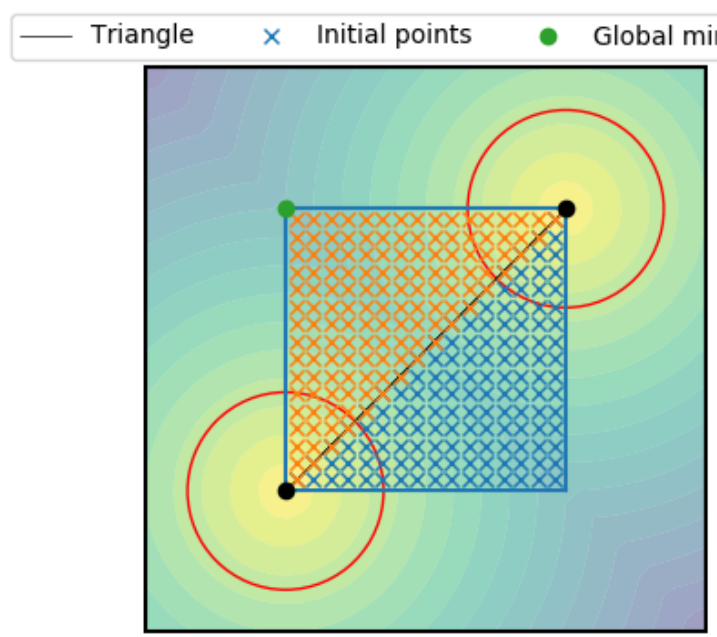

Figure 3. The algorithm divides the room into triangles and in each, calculates local optima (farthest points) from already determined control points. Subsequently, the farthest point is selected from this set of local optima and determined as the global minimum for the given iteration - the green point. At each iteration, a new background contour is calculated, which displays the distance from the control points in color.

Measurements shall be made at a given control point until the noise level exceeds a specified value. Subsequently, the measuring apparatus is moved to a new measuring point, and the entire process is repeated. Due to the lack of knowledge of the number of occurrences exceeding the specified noise level, the algorithm looks for the best distribution of control points in each iteration in order to cover as much room space as possible. The maximum distance is being looked from the already determined control points in the inner area of room $I$ :

$x_{n} \in \underset{x \in I}{\arg \max } \min _{i \in\{1, \ldots, n-1\}} d\left(x, x_{i}\right)$,

where $n$ is the iteration number and $x_{i}$ are control points positions selected in previous iterations.

The algorithm first determines the list of corners. Then one of the specified corners is selected randomly. The algorithm divides the floor plan of the room into individual triangles. In each triangle, the furthest local control point from the previously determined control points is calculated. Subsequently, these locally optimal solutions are compared to select the furthest point. This point is selected as a global optimum and is intended for noise level measurement. The entire process is shown in Figure 3; it is repeated until one of the conditions is satisfied:

1) There shall be at least one local point that satisfy the conditions specified in the standard (minimum distance from walls and other control points).

2) The noise measurement does not exceed the specified level at the measured control point.

3) The time interval for the measurement does not expire.

\section{EXPERIMENTS}

In order to improve the proposed algorithms for both purposes of measuring noise intensity, experiments were prepared to verify and test the algorithm. This section is organised as follows: the experimental environments are presented first, describing the rooms that were used in the verification; second, the control points are analysed for both algorithms; and third, the measurement time is analysed for both algorithms. The presented quantitative results demonstrate the performance of the proposed methods.
In the first experiment, the basic accuracy of calculations and the determination of control points were verified in a simple room layout for both algorithms. The size of the room was $4.0 \mathrm{~m} \times 4.0 \mathrm{~m}$. Each experiment is divided into two simulations: one for long-term and one for short-term noise. The simulations for the first experiment are shown in Figure 4.

The second experiment focused on a simulation of a more diverse room. The dimensions of the real room were measured and incorporated into the simulation. The results of the control point calculation for both algorithms are recorded in Figure 5.

The third experiment was focused on verifying the robustness of algorithms in more demanding conditions. An extensive
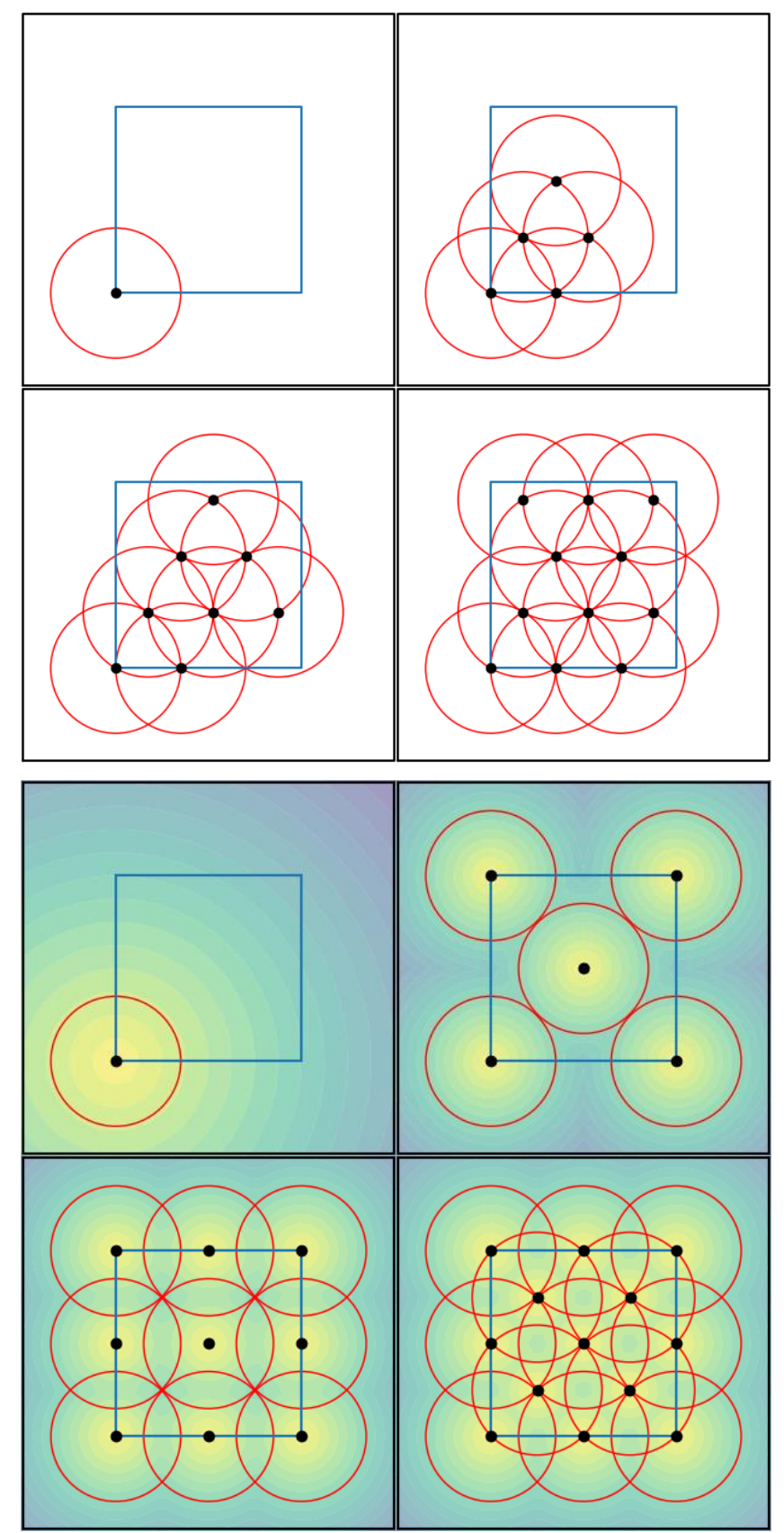

Figure 4. The first top four images show the first algorithm that determines the control points for long-term stationary noise. The algorithm gradually adds control points where the intersection of the $0.7 \mathrm{~m}$ boundary with the other boundaries (red circles) occurs. An algorithm created the lower quartet of the image for regular short-term noise. The algorithm focuses on covering the area as much as possible in each iteration. 

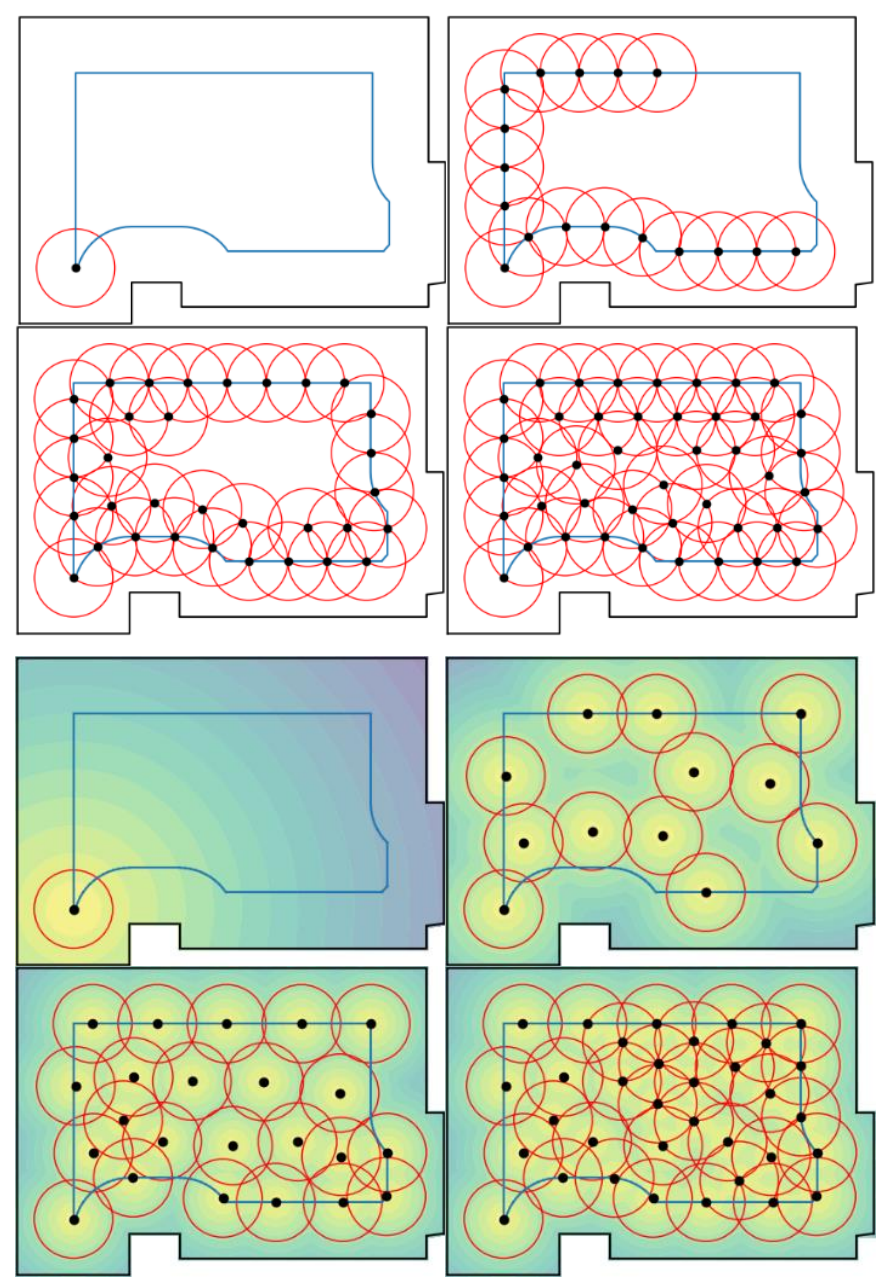

Figure 5 . The room is characterised by a more complex area where control points may occur. The results of the first algorithm are shown in the upper part. The figures show how the algorithm first determines the border control points in the marked area and then passes into the inner area of the room. The second algorithm created a network of control points, focusing on successively spaced control points throughout the area, as can be seen from the bottom figures.

segmented room was created, where the algorithms determined the control points (Figure 6).

The next experiments focused on rooms with internal obstacles, which in real conditions represent columns, partitions, etc. First, the algorithms were tested on simple rooms with one internal column in the area where control points are determined (Figure 7). According to the standard, $1 \mathrm{~m}$ has been defined around a fixed obstacle, where control points for noise measurement are not determined. In the last experiment, algorithms were tested for multiple obstacles (Figure 8).

\section{RESULT ANALYSIS}

This section presents the results of both algorithms, focusing on the number of checkpoints and the time required to determine them. Experiments were performed for simple, real, complex and built-up spaces. Both algorithms demonstrated functionality and robustness in the calculation of control points.

\subsection{Control points analysis}

An overview of the number of control points from both algorithms from individual experiments is reported in Table 1. Surprisingly, in the first experiment, the algorithm for long-term stationary noise found two control points less than the algorithm
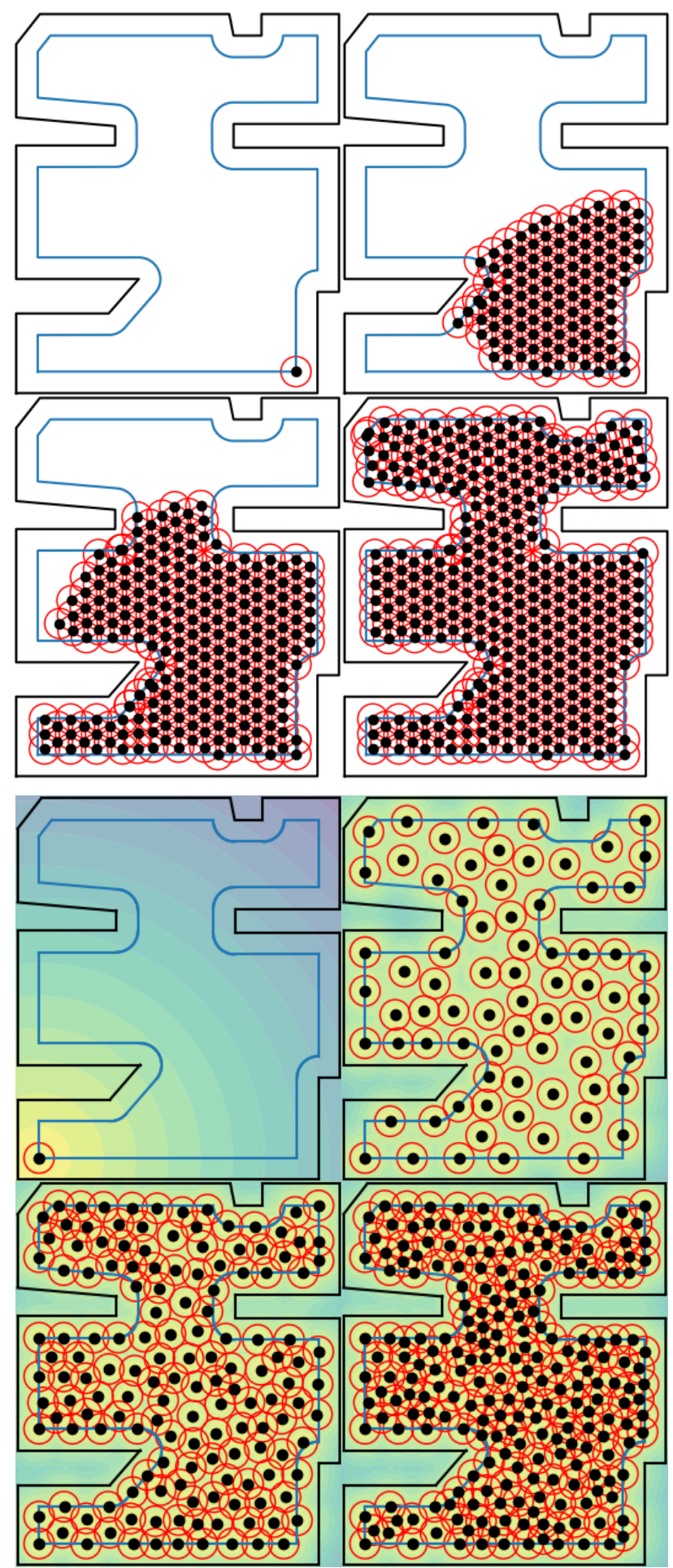

Figure 6. To test both algorithms in demanding conditions, a large room of intricate design was created. Both algorithms proceeded as expected in determining the control points. The results show that the algorithm for longterm stationary noise determined significantly more control points than the algorithm for regular short-term noise.

created for regular short-term noise. This anomaly occurred only in the first experiment, where it was an elementary room without a fragmented floor plan. Further testing showed that the algorithm for finding the maximum number of control points in the measuring area is not optimal and that it may not find the maximum number of control points for small rooms.

In the second experiment, the algorithm for long-term stationary noise discovered more control points than the second. 

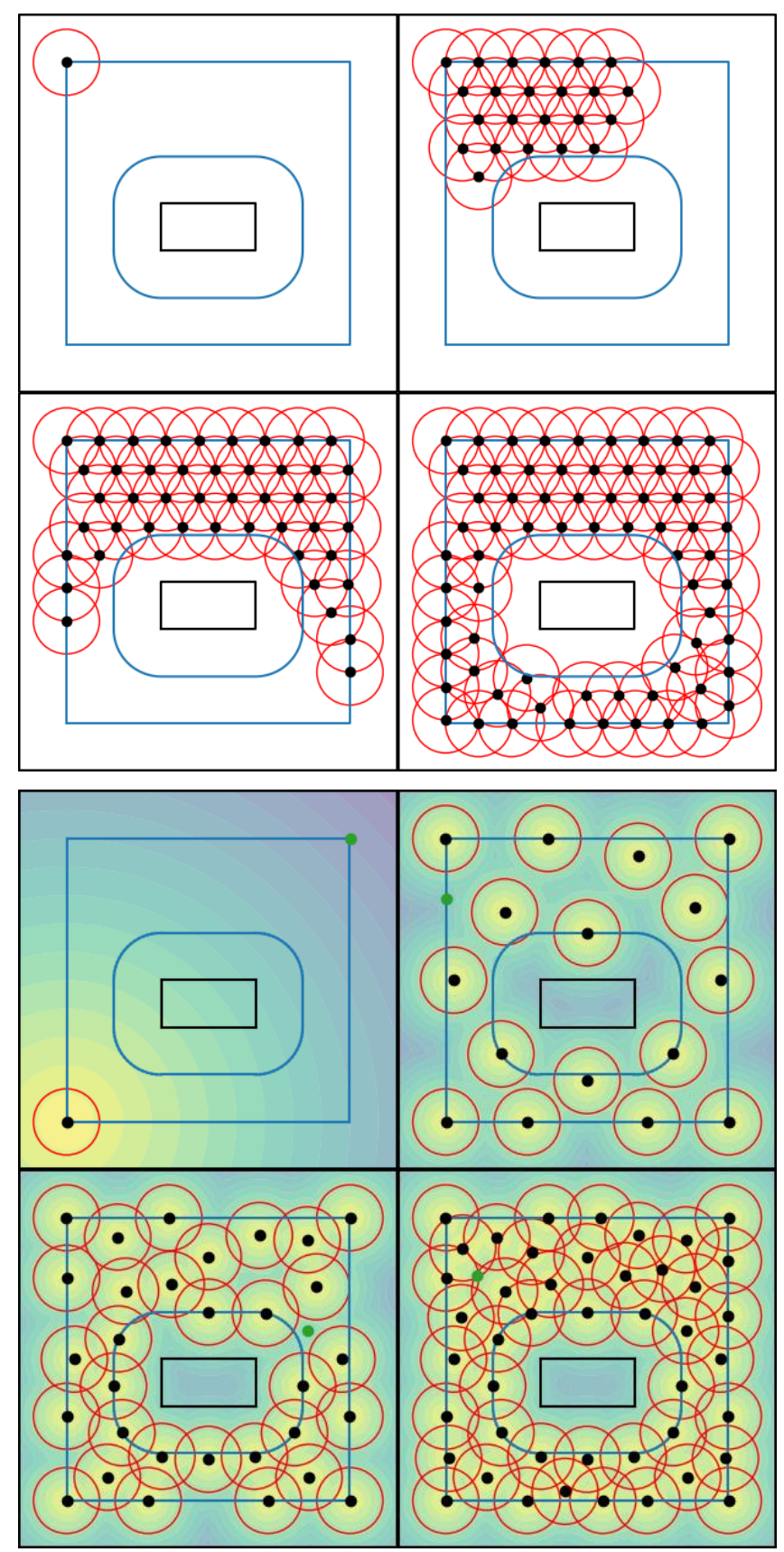

Figure 7. Another experiment was to test both algorithms in an environment where there is a simple barrier in the form of a column. Around this obstacle, both algorithms, as expected, created an area in which no measurement is performed.
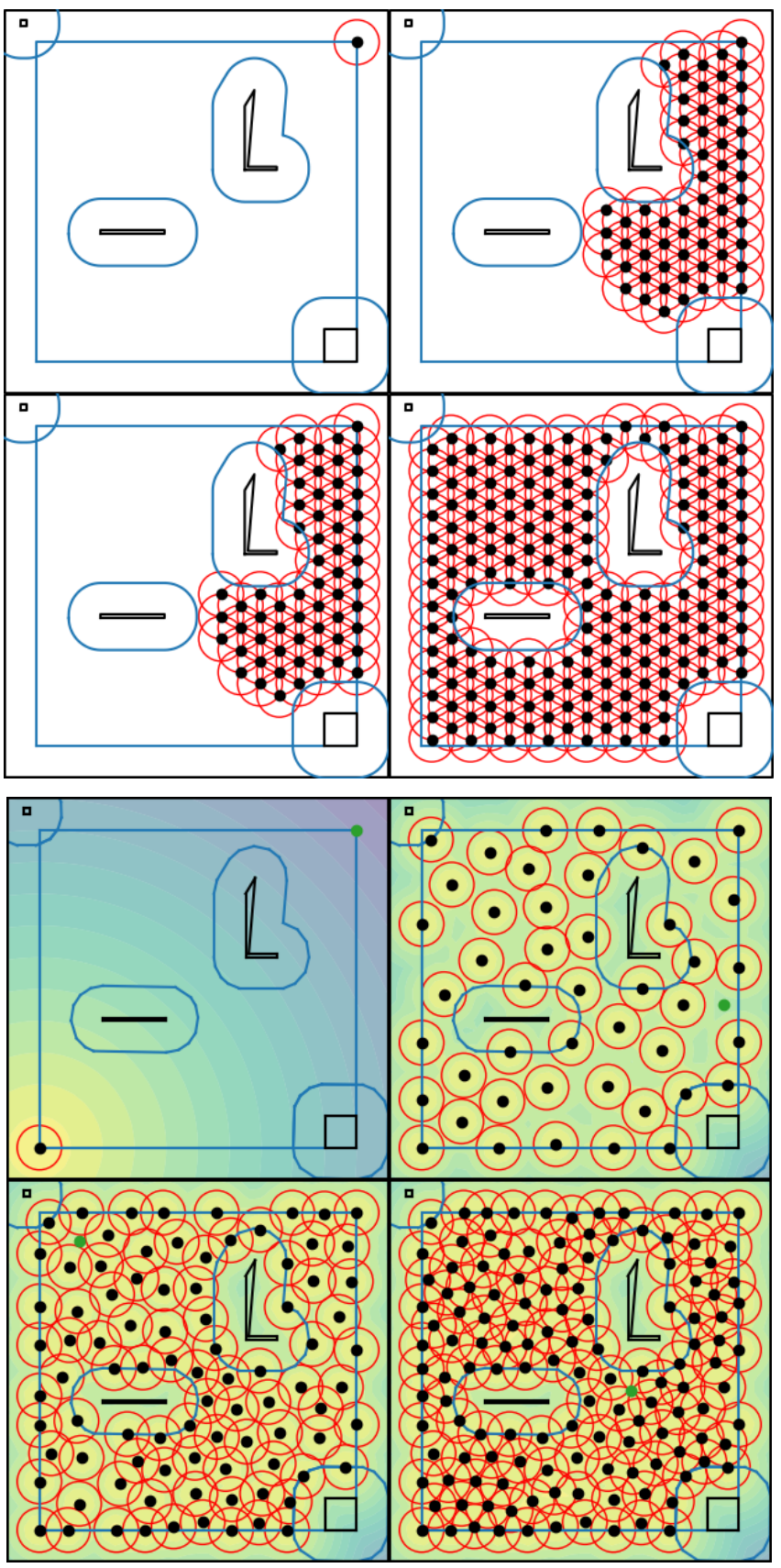

Figure 8. The last experiment focused on irregular, articulated obstacles that were both outside the measuring area and inside. Both algorithms proved their robustness and delineated the measuring area correctly.

Table 1. The total number of control points and computation time.

Number of control points

Room

Long-term
stationary noise

Sq. room (Figure 4)

Real room (Figure 5)

Large room (Figure 6)

Sq. room with column (Figure 7)

Sq. room with obstacles (Figure 8)

$\begin{array}{cc}11 & 13 \\ 44 & 33 \\ 360 & 224 \\ 69 & 47 \\ 187 & 133\end{array}$

\begin{tabular}{c}
$\begin{array}{c}\text { Short-term } \\
\text { recurring noise }\end{array}$ \\
\hline 13 \\
33 \\
224 \\
47 \\
133
\end{tabular}

Computation time [min] 
In the third experiment, the algorithm for long-term stationary noise determined 360 control points in the final result. The algorithm for regular short-term noise identified 224 control points. Obstacles were inserted into the measured area in the fourth and fifth experiments. The difference in the number of control points for larger rooms shows that the first algorithm is indeed more suitable for long-term noise.

\subsection{Estimating the measurement time}

The algorithms differ according to the measurement purpose and the time they need to determine the control points. In all experiments, the time required to determine all the control points was recorded and is presented in Table 1.

Results show that the algorithm for the first purpose calculates all control points much faster than the algorithm for regular short-term noise. In the third and the fifth experiment, the most apparent difference in the time calculation of control points was between the two algorithms. In actual measurements, however, this difference is not decisive. Vice versa, when measuring longterm stationary noise, a faster determination of other control points is needed - changing the measuring point sets immediately after recording the noise level at that point. For regular shortterm noise, the algorithm has enough time to calculate. The change of the measuring point is not known, and the noise level at the given control point is expected to be exceeded. While waiting for the noise level to be exceeded, the algorithm can determine the next control points based on the dimensions of the room. Optionally, the algorithms can compute control points offline before the measurement based on the floor plan measured either manually or automatically using Lidar.

\section{CONCLUSIONS}

This paper aimed to create algorithms for two specific purposes of noise measurement. The first algorithm was developed to determine control points for long-term stationary noise, and it finds the maximum number of control points in the room. The second algorithm was created to determine control points for regular short-term noise. For this purpose, the program does not know the number of iterations of the measurement. Therefore, it looked for the location of the following control point in the measuring area to cover as large an area as possible.

Both algorithms were tested in different rooms during the experiments, from simple floor plans over large rugged rooms to the room with obstacles. The simulations showed that the proposed repeatable algorithms satisfy the specified conditions set by the standard. The time required to determine all control points in the defined area during the simulations for both algorithms was recorded.

The novelty of the noise measurement methodology consists in the design and testing of both algorithms, with the control points being determined based on the dimensions of the room and the purpose of the measurement. The control points were determined to meet the conditions based on the standard. If this algorithm is used in the future to a mobile robotic unit that will contain a measuring device, the measurement can take place entirely autonomously without the presence of an operator.

Therefore, we decided to test both of our algorithms using a robotic unit that will initially measure the floor plan by simultaneous localisation and mapping in the next phase. The knowledge published in [20] can also be used to navigate the room with the robotic unit. The constructed map is used to compute control points, and the robotic platform measures these points afterwards.

\section{ACKNOWLEDGEMENT}

This work was supported by the Grant Agency of the Czech Technical University in Prague, Grant No. SGS20/070/OHK3/1T/13.

\section{REFERENCES}

[1] S. S. Carlisle, The role of measurement in the development of industrial automation, Acta IMEKO 3 (2014) 1, pp. 4-9. DOI: $\underline{10.21014 / \text { acta imeko.v3i1.190 }}$

[2] J. Svatos, J. Holub, T. Pospisil, A measurement system for the long-term diagnostics of the thermal and technical properties of wooden houses, Acta IMEKO 9 (2020) 2, pp. 3-10. DOI: $10.21014 /$ acta imeko.v9i3.762

[3] International Organization for Standardization (ISO), 1996 2:2017 Acoustics - Description, measurement and assessment of environmental noise - Part 2: Determination of sound pressure levels, ISO, Geneva, Switzerland, 2017

[4] C. S. Institute, "1996-2 Acoustics - Description, measurement and assessment of environmental noise - Part 2: Determination of sound pressure levels", Czech Standards Institute, 2018

[5] M. Genesca, J. Romeu, T. Pamies, A. Sanchez, Aircraft noise monitoring with linear microphone arrays, IEEE Aerospace and Electronic Systems Magazine 25(1) (2010), pp. 14-18. DOI: $10.1109 /$ MAES.2010.5442149

[6] J.W. Pak, M.K. Kim, Convolutional neural network approach for aircraft noise detection, Proc. of IEEE 2019 International Conference on Artificial Intelligence in Information and Communication (ICAIIC), Okinawa, Japan, 11-13 February 2019, pp. $430-434$.

DOI: $10.1109 /$ ICAIIC.2019.8669006

[7] C. Asensio, M. Ruiz, M. Recuero, G. Moschioni, M. Tarabini, A novel intelligent instrument for the detection and monitoring of thrust reverse noise at airports, Acta IMEKO 4 (2015) 1, pp. 5-10. DOI: $10.21014 /$ acta imeko.v4i1.154

[8] X. Chen, D. Wang, X. Yu, Z.D. Ma, Analysis and control of automotive interior noise from powertrain in high frequency, Proc. of 2009 IEEE Intelligent Vehicles Symposium, Xi'an, China, 3-5 June 2009, pp. 1334-1339. DOI: $\underline{10.1109 / \text { IVS.2009.5164478 }}$

[9] International Electrotechnical Commission, IEC 61400-11 Wind turbines - Part 11: Acoustic noise measurement techniques, 3.1 Edition, pp.1-131, June 2018

[10] IEEE Instrumentation and Measurement Society, IEEE Standard for Wind Turbine Aero Acoustic Noise Measurement Techniques, IEEE Std 2400-2016, pp.1-24, 1 July 2016. DOI: 10.1109 /IEEESTD.2016.7502056

[11] S. Janhunen, A. Grönman, K. Hynynen, M. Hujala, M. Kuisma, P. Härkönen, Audibility of wind turbine noise indoors: Evidence from mixed-method data, Proc. of 2017 IEEE 6th International Conference on Renewable Energy Research and Applications (ICRERA), San Diego, CA, USA, 5-8 Nov. 2017, pp. 164-168. DOI: $10.1109 /$ ICRERA.2017.8191260

[12] G. Graziuso, M. Grimaldi, S. Mancini, J. Quartieri, C. Guarnaccia, Crowdsourcing data for the elaboration of noise maps: a methodological proposal, Journal of Physics: Conference Series. 1603 (2020), art. 012030. DOI: $\underline{10.1088 / 1742-6596 / 1603 / 1 / 012030}$

[13] C. Buratti, Indoor noise reduction index with open window, Applied Acoustics, 2002, pp. 431-451.

[14] C. Guarnaccia, J. Quartieri, A. Ruggiero, Acoustical noise study of a factory: Indoor and outdoor simulations integration procedure, International Journal of Mechanics 8(1) (2014), pp 298-306. Online [Accessed 13 August 2021] https://www.naun.org/main/NAUN/mechanics/2014/a162003 -065.pdf 
[15] S. Pujol, M. Berthillier, J. Defrance, J. Lardiès, R. Petit, H. Houot, J. P. Levain, C. Masselot, F. Mauny, Urban ambient outdoor and indoor noise exposure at home: A population-based study on schoolchildren, Applied Acoustics 73(8) (2012), pp 741-750. DOI: $10.1016 / \mathrm{j}$.apacoust.2012.02.007

[16] P. H. T. Zannin, F. Ferraz, Assessment of indoor and outdoor noise pollution at a university hospital based on acoustic measurements and noise mapping, Open Journal of Acoustics 4(6) (2016), pp 71-85. DOI: $10.4236 /$ oja.2016.64006

[17] B. Locher, A. Piquerez, M. Habermacher, M. Ragettli, M. Röösli, M. Brink, C. Cajochen, D. Vienneau, M. Foraster, U. Müller, J. M. Wunderli, Differences between outdoor and indoor sound levels for open, tilted, and closed windows. International Journal of Environmental Research and Public Health 15(1) (2018), art. 149. DOI: $\underline{10.3390 / \text { ijerph15010149 }}$
[18] M. Bonomolo, P. Ribino, C. Lodato, G. Vitale, Post occupancy evaluation and environmental parameters monitoring by a humanoid robot, Proc. of 2019 IEEE International Conference on Environment and Electrical Engineering and 2019 IEEE Industrial and Commercial Power Systems Europe (EEEIC I\&CPS Europe), Genova, Italy, 11-14 June 2019, pp 1-6. DOI: $10.1109 /$ EEEIC.2019.8783688

[19] E. Martinson, R. C. Arkin, Noise maps for acoustically sensitive navigation, Proceedings Volume 5609, Mobile Robots XVII, 2004, pp. 50-60.

DOI: $10.1117 / 12.581461$

[20] D. Fontanelli, M. David, R. Tizar, A fast and low-cost vision-based line tracking measurement system for robotic vehicles, Acta IMEKO 4 (2015) 2, pp. 90-99.

DOI: $10.21014 /$ acta imeko.v4i2.245 